\section{(2) OPEN ACCESS}

\title{
Meaning in life and quality of life: palliative care patients versus the general population
}

\section{Mathieu Bernard (10 , ${ }^{1}$ André Berchtold, ${ }^{2}$ Florian Strasser, ${ }^{3}$ Claudia Gamondi (D) ,", Gian Domenico Borasio ${ }^{1}$}

${ }^{1}$ Palliative and Supportive Care Service, Lausanne University Hospital and University of

Lausanne, Lausanne, Switzerland ${ }^{2}$ Institute of Social Sciences \& NCCR LIVES, University of Lausanne, Lausanne, Switzerland ${ }^{3}$ Department of Internal Medicine and Palliative Care Center, Clinical Medical Oncology \& Hematology, Cantonal Hospital, St. Gallen, Switzerland ${ }^{4}$ Palliative Care Clinic, Oncology Institute of Southern switzerland, Palliative Care Departement, Bellinzona, Ticino, Switzerland

\section{Correspondence to}

Dr Mathieu Bernard, Palliative and Supportive Care Service, University of Lausanne, Lausanne, Switzerland; mathieu.bernard@chuv.ch

Received 21 January 2020 Revised 30 April 2020 Accepted 21 May 2020

\section{Check for updates}

(C) Author(s) (or their employer(s)) 2020. Re-use permitted under CC BY-NC. No commercial re-use. See rights and permissions. Published by BMJ.

\footnotetext{
To cite: Bernard $\mathrm{M}$, Berchtold A, Strasser F, et al. BMJ Supportive \& Palliative Care Epub ahead of print: [please include Day Month Year]. doi:10.1136/ bmjspcare-2020-002211
}

\section{ABSTRACT}

Background and objectives Meaning in life (MIL) represent a key topic in palliative care. The aims of this study were to explore (1) the differences in perceived MIL and in the meaning-relevant life areas between a representative sample of the Swiss population and palliative care patients, and (2) to what extent MIL can be considered as a significant predictor of quality of life (QOL).

Methods A cross-sectional study was conducted separately for the patients (faceto-face interviews) and the general population (telephone survey). MIL was measured with the Schedule for Meaning in Life Evaluation (SMILE) and QOL with a single-item visual analogue scale (0-10). Sociodemographic variables were controlled for in the analyses.

Results 206 patients and 1015 participants from the Swiss population completed the protocol. Results indicated high MIL scores in both populations even if the difference was significant (patients 81.9 vs general population $87, p<0.001)$. Patients were more likely to cite 'family' (OR=1.78), 'social relations' $(\mathrm{OR}=1.9)$, 'spirituality and religion' ( $\mathrm{OR}=3.93)$, 'social commitment' (OR=1.94) and 'growth' $(\mathrm{OR}=2.07)$, and less likely to cite 'finances' $(\mathrm{OR}=0.15)$ and 'health' (OR=0.21) as MILrelevant areas. The SMILE scores and MIL areas explained $21.8 \%$ of the QOL variance for the patients and $15.1 \%$ for the representative sample.

Conclusions Our data emphasise the importance of MIL as a contributor to QOL in both populations. It highlights the importance of the life areas contributing to MIL, especially social interactions for both populations, and spirituality and areas related to growth in palliative care patients.

\section{INTRODUCTION}

The concept of quality of life (QOL) is the central outcome measure in palliative care research. The WHO defines QOL as 'the individual perception of the position in life in the context of the culture and value system in which they live, and in relation to their goals, expectations, standards and concerns'. In palliative care research, focusing on a general individual perception of QOL by using a single-item question is still uncommon. Many instruments used for research were designed using either a health-related concept of $\mathrm{QOL}^{2}$ or a multidimensional perspective. ${ }^{3}{ }^{4}$ Studies have shown in recent years that QOL at the end of life is linked to non-physical determinants. ${ }^{56}$

Among these, the spiritual domain is relevant for palliative patients. ${ }^{3}$

A paper of the European Association for Palliative Care Task Force on spiritual care defines spirituality as the dynamic dimension of human life that relates to the way persons (individual and community) experience, express and/or seek meaning, purpose and transcendence, and the way they connect to the moment, to self, to others, to nature, to the significant and/or the sacred'. Meaning in life (MIL) represents a core element of spirituality, and loss of MIL represents an important clinical issue. It has been associated with important outcomes in the end of life context: anxiety and depression, ${ }^{8}$ suicidal ideas, ${ }^{9}$ the desire for hastened death ${ }^{10}$ and various physical symptoms. ${ }^{11}$

From a clinical and research point of view, meaning and MIL have been widely discussed in the psychology literature. The meaning-making model explains how people adjust themselves by giving meaning to various stressful life events, as is the case with a life-threatening illness. ${ }^{12}$ Several manualised interventions were developed in order to help people facing 
a loss of meaning. ${ }^{13}{ }^{14}$ Victor Frankl, a pioneer in the development of psychological interventions centred on MIL, considered it as an individual construct, difficult to capture from an external standpoint. The individual identification of life areas considered as sources of MIL represents a crucial point when initiating a therapeutic process. ${ }^{15}$ The Schedule for Meaning in Life Evaluation (SMILE) allows individuals to choose freely the life areas that they consider important for their own MIL. ${ }^{16}$

We have previously reported separately data obtained with the SMILE in a representative sample of the Swiss population and a sample of Swiss palliative care patients. ${ }^{5}{ }^{17}$ Relating a patient population with the general population may help to take a step back by contrasting what is normal from a developmental perspective and what is specific to a lifethreatening disease situation. Identifying areas of life that may contribute to QOL can also provide interesting insights for interventions. In analogy to the study of Fegg et al, ${ }^{18}$ we compare the two populations regarding MIL, but we go further by exploring to what extent MIL can predict QOL and what is the discrepancy between these populations.

\section{METHODS}

The data for this comparison study using an exploratory, cross-sectional design were collected separately for the palliative care patients and the representative sample of the Swiss population. Collection methods and part of the results have been published. ${ }^{517}$

Data from the general population in Switzerland were collected through a telephone survey performed by a professional company between November and December 2013, resulting in a representative sample across the three linguistic regions, age and sociodemographic characteristics. In the first step, households were drawn and the sampling was stratified by linguistic region and town size. The selected households were then called on the phone and the interviewer established their composition. The selection of the respondent among the household members happened by quota (sex-age interlocked). All interviewers of the survey company were trained by two study investigators and received a standardised protocol, including an assessment of MIL, QOL, health status and sociodemographic data: gender, age, education, employment, marital status, profession, self-evaluation of the socioeconomic status, residence (population size) and linguistic region.

For palliative care patients, a cross-sectional study, based on structured face-to-face interviews, was conducted in different palliative care contexts of the three Swiss linguistic regions (German, French and Italian) between 2012 and 2015. Inclusion criteria were (1) 18 years or older, (2) being treated by one of the specialised palliative care teams involved and $(3)<6$ months life expectancy according to the treating physician. Exclusion criteria were (1) psychiatric disease, (2) significant cognitive impairment and (3) insufficient knowledge of the local language. Patients hospitalised in a palliative care unit or cared for by a palliative home care team who fulfilled the inclusion criteria were asked to participate in the study by a research collaborator who was not part of the palliative care team. After obtainment of informed consent, a meeting was scheduled with the research collaborator in order to complete the case report form assessing MIL, QOL, religiosity, psychological distress and attitudes towards hastened death.

For this specific study including both populations, assessment of MIL, QOL and sociodemographic data (gender, age, education, employment, marital status, residence and linguistic region) were considered.

\section{Instruments}

Schedule for MIL evaluation

This instrument was developed and validated by Fegg et al. ${ }^{16}$ Respondents are first asked to indicate three to seven areas that provide meaning to their life. The relevant areas contributing to MIL were classified in 1 of 15 categories reported in a specific manual developed for this instrument (family, partnership, social relations, occupation/work, leisure time/relaxation, home and garden, finances, spirituality/religion, health, satisfaction, nature/animals, social commitment, hedonism, art and culture, and growth (see Simmons et $a l^{6}$ and www.psychotherapie-muenchen.de/downloads/SMiLE_Manual.pdf).

The importance and the current level of satisfaction associated with each area are rated on Likert scales (seven points, from -3 to +3 , for the satisfaction, and eight points, from 0 to 7 , for the importance). An 'index of weighting' (IoW, range 0-100) and an 'index of satisfaction' (IoS, range 0-100) indicate the mean weighing score and the mean satisfaction score for the areas mentioned. A SMILE total score, 'index of weighted satisfaction' (IoWS, 0-100), indicates the global MIL satisfaction.

Subjective Single-Item QOL

In both groups, subjective QOL was measured after completion of the SMILE questionnaire with the Single-Item Quality of Life Scale-Numeric Rating Scale $0-10$, ranging from 0 (worst imaginable QOL) to 10 (best imaginable QOL). This approach to measuring QOL was validated by de Boer et al and Idler and Benyamini. ${ }^{19} 20$

\section{Statistics}

Differences between sociodemographic characteristics of the two samples were assessed using the $\chi^{2}$ test. The mean of the different SMILE scores was computed in both samples, and linear regression models were used to determine whether sample membership could explain 
the scores, controlling for sociodemographic factors. The distribution of MIL areas cited by the respondents was computed separately for each sample. A logistic regression model was computed to assess the presence of each MIL area in function of the sample membership.

After having applied bivariate analyses ( $t$-test and correlation), we built models explaining the subjective QOL from the SMILE scores. We began by considering the full sample, and then we built separate models for the patient and the general population samples. In each case, we proceeded in two steps: in the first one, we used only the three main scores of the SMILE as predictors (IoS, IoW and IoWS). In the second one, we also included indicator variables of the presence or not of the 15 different MIL areas, and we applied a backward selection procedure in order to identify the best model. All models were controlled for sociodemographic characteristics (age, gender, marital status and education). Controlling for sociodemographic variables allows keeping them constant and avoiding any influence on the dependent variable.

Sample size was estimated based on the planned use of multivariate analyses. According to the literature, 20 observations are needed for each independent variable considered in the regression analyses. Given the very small number of missing data in the sociodemographic characteristics $(n=9$ for the marital status and $n=2$ for education on 1221 participants), no special processing was carried out and all calculations were done with pairwise deletion. The number of missing data concerning MIL and QOL was also very small $(\mathrm{n}=9$ for QOL and $\mathrm{n}=6$ for MIL), and these participants were not considered in the regression analyses.

The type I error was set to 0.05 for all inferential computations. Statistical analyses were performed using the Statistical Package for the Social Sciences V.24.

\section{RESULTS}

\section{Recruitment and descriptive results}

For the Swiss population, 6671 telephonic contacts were realised, and 1015 (15\%) individuals completed all questionnaires (405 French, 400 German and 210 Italian participants; see ref. 18 for details). Regarding the patients sample, in the three Swiss linguistic regions, 588 participants met the inclusion criteria, 206 participants gave their consent and completed the questionnaires (77 French, 89 German, and 40 Italian patients; see Bernard et $a l^{5}$ for details).

Table 1 gives the sociodemographic and medical characteristics of the two populations. Results showed significant differences between the two populations regarding age, marital status and education level. However, effect sizes can be considered as small, given the Cramer's V (between 0.016 and
0.180). Cancer was the most common diagnosis among the palliative care patients.

Differences in MIL between palliative care patients and the Swiss sample

Results of the linear regressions indicated significant differences between the two populations regarding the IoS, the index of importance (IoW) and the SMILE total score (IoWS), with lower scores in the patients sample. On the other hand, no difference was found regarding the number of cited areas.

Differences in sources of MIL between palliative care patients and the Swiss sample

'Family' and 'social relations' were the two most cited areas in both populations $(76 \%$ for family in the two populations and $49 \%$ vs $39 \%$ for the social relations in the patients and the Swiss sample, respectively). The relational dimension represents the most important dimension for MIL in the two populations. The results of the logistic bivariate regression model show that patients were significantly more likely to cite family $(\mathrm{OR}=1.78)$, social relations $(\mathrm{OR}=1.9)$, 'spirituality and religion' $(\mathrm{OR}=3.93)$, 'social commitment' $(\mathrm{OR}=1.94)$ and 'growth' $(\mathrm{OR}=2.07)$, and less likely to cite 'finances' $(\mathrm{OR}=0.15)$ and 'health' $(\mathrm{OR}=0.21)$ (see table 3$)$.

Notice that the apparent inconsistency between the percentages and the OR for the family category is due to the fact that the regression model was controlled for sociodemographic factors, which was not the case for the percentages. In particular, there were significant associations between marital status, age and civil status.

\section{MIL as a QOL predictor}

The results of the independent samples t-test indicated a significant difference in QOL between the palliative care patients and the Swiss sample $(\mathrm{M}=5.03$, $\mathrm{SD}=2.6 ; \mathrm{M}=7.56, \mathrm{SD}=1.7$, respectively; $\mathrm{t}=17.549$, $\mathrm{p}=0.000$ ). The correlation values between $\mathrm{QOL}$ and the SMILE scores indicated that these two dimensions were not independent and justified the use of linear regressions to test in which measure MIL was associated with QOL (Pearson correlation values were between 0.220 and $0.364, \mathrm{p}=0.000$ ).

The differences between the two populations clearly warranted a separate analysis to obtain a better identification of the QOL predictors. Therefore, we performed regression analyses on the two samples separately.

\section{Palliative care patients}

A first linear regression model (see top of table 4), including the three scores of the SMILE, showed that $13 \%$ of the QOL variance (adjusted $\mathrm{R}^{2}$ ) was explained, but no predictor reached significance. 
Table 1 Demographic characteristics in each sample and main diagnosis of the palliative care patients

\begin{tabular}{|c|c|c|c|c|c|c|c|c|}
\hline \multirow[b]{2}{*}{ Variables } & \multicolumn{2}{|c|}{ Patients } & \multicolumn{2}{|c|}{ Swiss sample } & \multicolumn{2}{|l|}{ Total } & \multirow[b]{2}{*}{ P value } & \multirow[b]{2}{*}{ Cramer's V } \\
\hline & $\mathrm{n}$ & $\%$ & $n$ & $\%$ & $\mathrm{n}$ & $\%$ & & \\
\hline Age (years) & & & & & & & $<0.001$ & 0.261 \\
\hline $16-19$ & 0 & 0.0 & 42 & 4.1 & 42 & 3.4 & & \\
\hline $20-29$ & 0 & 0.0 & 99 & 9.8 & 99 & 8.1 & & \\
\hline $30-39$ & 1 & 0.5 & 99 & 9.8 & 100 & 8.2 & & \\
\hline $40-49$ & 12 & 5.8 & 144 & 14.2 & 156 & 12.8 & & \\
\hline $50-59$ & 38 & 18.4 & 121 & 11.9 & 159 & 13.0 & & \\
\hline $60-69$ & 65 & 31.6 & 244 & 24.0 & 309 & 25.3 & & \\
\hline $70-79$ & 58 & 28.2 & 167 & 16.5 & 225 & 18.4 & & \\
\hline $80-89$ & 28 & 13.6 & 88 & 8.7 & 116 & 9.5 & & \\
\hline $90-99$ & 4 & 1.9 & 11 & 1.1 & 15 & 1.2 & & \\
\hline Total & 206 & 100.0 & 1015 & 100 & 1221 & 100.0 & & \\
\hline Gender & & & & & & & 0.575 & 0.016 \\
\hline Male & 100 & 48.5 & 471 & 46.4 & 571 & 46.8 & & \\
\hline Female & 106 & 51.5 & 544 & 53.6 & 650 & 53.2 & & \\
\hline Total & 206 & 100.0 & 1015 & 100.0 & 1221 & 100.0 & & \\
\hline Marital status & & & & & & & $<0.001$ & 0.180 \\
\hline Single & 31 & 15.4 & 209 & 20.7 & 240 & 19.8 & & \\
\hline Married/legal partnership & 91 & 45.3 & 597 & 59.0 & 688 & 56.8 & & \\
\hline Divorced/separated & 45 & 22.4 & 92 & 9.1 & 137 & 11.3 & & \\
\hline Widowed & 34 & 16.9 & 113 & 11.2 & 147 & 12.1 & & \\
\hline Missing & 5 & 2.4 & 4 & 0.4 & 9 & 0.7 & & \\
\hline Total & 201 & 100.0 & 1011 & 100.0 & 1212 & 100.0 & & \\
\hline Education & & & & & & & $<0.001$ & 0.149 \\
\hline Primary school & 44 & 21.6 & 174 & 17.1 & 218 & 17.9 & & \\
\hline Professional diploma & 100 & 49.0 & 432 & 42.6 & 532 & 43.6 & & \\
\hline Secondary school & 5 & 2.5 & 102 & 10.0 & 107 & 8.8 & & \\
\hline Highschool & 30 & 14.7 & 92 & 9.1 & 122 & 10.0 & & \\
\hline University & 24 & 11.8 & 208 & 20.5 & 232 & 19.0 & & \\
\hline Other & 1 & 0.5 & 7 & 0.7 & 8 & 0.7 & & \\
\hline Missing & 2 & 1 & & & & 0.2 & & \\
\hline Total & 204 & 100.0 & 1015 & 100.0 & 1219 & 100.0 & & \\
\hline \multicolumn{9}{|l|}{ Region } \\
\hline French & 77 & 37.4 & 405 & 39.9 & 482 & 39.5 & 0.598 & 0.029 \\
\hline German & 89 & 43.2 & 400 & 39.4 & 489 & 40.0 & & \\
\hline Italian & 40 & 19.4 & 210 & 20.7 & 250 & 20.5 & & \\
\hline Total & 206 & 100.0 & 1015 & 100.0 & 1221 & 100.0 & & \\
\hline \multicolumn{9}{|l|}{ Primary diagnosis } \\
\hline Cancer & 197 & 95.6 & & & & & & \\
\hline Heart disease & 1 & 0.5 & & & & & & \\
\hline Pulmonary disease & 1 & 0.5 & & & & & & \\
\hline ALS & 5 & 2.4 & & & & & & \\
\hline Missing & 2 & 1.0 & & & & & & \\
\hline Total & 206 & 100 & & & & & & \\
\hline
\end{tabular}

ALS, amyotrophic lateral sclerosis.

A second linear regression model (see bottom of table 4), using the backward selection process with the 15 MIL areas in addition to the SMILE scores, explained $22 \%$ of the QIL variance. The results showed a positive and significant association with the index of importance (IoW) of the SMILE, and also the 'satisfaction' and the social commitment areas.

\section{Swiss sample}

The first linear regression model (see top of table 5), including the three scores of the SMILE, explained
$13 \%$ (adjusted $\mathrm{R}^{2}$ ) of the QOL variance, with a positive significant association with the index of importance (IoW) of the SMILE. A second linear regression model (see bottom of table 5), using the backward selection process with the 15 MIL areas, in addition to the SMILE scores, explained 15\% of the QOL variance. The results showed a positive and significant association with the index of importance (IoW) and the total score (IoWS) of the SMILE, and also the satisfaction and the social relations areas. 
Table 2 Descriptive characteristics of the different SMILE scores in both samples and linear regression models predicting these scores from group membership (patient vs Swiss sample, the Swiss sample being the reference)

\begin{tabular}{|c|c|c|c|c|c|c|}
\hline \multirow[b]{2}{*}{ SMILE indices } & \multirow{2}{*}{$\begin{array}{l}\text { Patients } \\
\text { Mean (SD) }\end{array}$} & \multirow{2}{*}{$\begin{array}{l}\text { Swiss sample } \\
\text { Mean (SD) }\end{array}$} & \multirow{2}{*}{$\begin{array}{l}\text { Total } \mathrm{R}^{2} \\
\text { adjusted }\end{array}$} & \multirow[b]{2}{*}{ B } & \multirow{2}{*}{$\begin{array}{l}\text { Beta } \\
\text { standardised }\end{array}$} & \multirow[b]{2}{*}{$P$ value } \\
\hline & & & & & & \\
\hline loW & $81.0(14.3)$ & $82.8(12.5)$ & 0.030 & -2.230 & -0.064 & 0.029 \\
\hline los & $79.9(17.6)$ & $86.1(13.4)$ & 0.044 & -6.715 & -0.173 & $<0.001$ \\
\hline loWS & $81.9(17.2)$ & $87.0(13.5)$ & 0.033 & -5.463 & -0.142 & $<0.001$ \\
\hline Number of areas mentioned & $4.0(1.5)$ & $4.1(1.4)$ & 0.000 & -0.059 & -0.016 & 0.584 \\
\hline
\end{tabular}

Regression models are controlled for sociodemographic variables: age, gender, marital status and education.

IOS, index of satisfaction; IoW, index of weighting; loWS, index of weighted satisfaction; SMILE, Schedule for Meaning in Life Evaluation.

\section{DISCUSSION}

To the best of our knowledge, this study is only the second one that aimed to explore differences in MIL between a palliative care population and a representative sample of a population from a national survey, after the study of Fegg et al. ${ }^{18}$ The latter study took place in Germany, a more homogeneous sociocultural context than Switzerland with its mix of German and Latin populations. A novel purpose of our study was to explore the relationship between MIL and the QOL in these two populations.

\section{Level of satisfaction in MIL: meaning-making hypothesis}

The high levels reported in the SMILE total scores in both populations $(>80)$ are comparable with those found in other studies, including different kind of populations: patients with cancer, ${ }^{21}{ }^{22}$ palliative care patients ${ }^{2324}$ and general populations. ${ }^{1825}$ This propensity towards high scores is also apparent in a study with Indian palliative care patients. ${ }^{26}$ Fegg et $a l^{16}$ already mentioned a ceiling effect of the SMILE to explain the small difference between the samples. At first sight, these scores in MIL appear to be surprising, given the end-of-life context. However, the prevalence of mood disorders is about $30 \%$ in the oncological, palliative care and haematological settings. ${ }^{27}$ This indicates that a majority of patients are able to adapt successfully to the constraints of an end-of-life situation.

Another explanation is the response shift phenomenon, which refers to a change in values, goals and expectations towards the remaining life. ${ }^{28}$ Such a transformation process may occur, consciously or subconsciously, when people encounter adverse experiences during their lives. The announcement of a potential life-threatening illness and the subsequent confrontation with one's own death can certainly be viewed as a one of 'those events that have seismic impact on the individual assumptive world'. ${ }^{29}$ A growing body of evidence exists on the psychological mechanisms employed by individuals in order to cope with adversity and difficulties (eg, see the review of Windle ${ }^{30}$ on resilience).

Table 3 Percentage of participants listing each MIL area category in each sample and logistic regression model predicting the presence of each MIL area from group membership (patient vs Swiss sample (reference category))

\begin{tabular}{|c|c|c|c|c|c|c|c|}
\hline & $\begin{array}{l}\text { Patients } \\
(n=206) \\
\%\end{array}$ & $\begin{array}{l}\text { Swiss sample } \\
(\mathrm{n}=1015) \\
\%\end{array}$ & $\begin{array}{l}\text { OR } \\
\text { Exp (B) }\end{array}$ & $95 \% \mathrm{Cl}$ & & B & $P$ value \\
\hline Family & 76.7 & 76.3 & 1.783 & 1.188 & 2.676 & 0.578 & 0.005 \\
\hline Partnership & 18.0 & 16.4 & 1.468 & 0.949 & 2.270 & 0.384 & 0.084 \\
\hline Social relation & 49.0 & 39.1 & 1.898 & 1.365 & 2.641 & 0.641 & $<0.001$ \\
\hline Occupation and work & 24.8 & 41.5 & 0.748 & 0.505 & 1.108 & -0.290 & 0.148 \\
\hline Leisure time & 33.0 & 37.7 & 0.872 & 0.617 & 1.232 & -0.137 & 0.438 \\
\hline Home and garden & 10.2 & 7.5 & 1.087 & 0.636 & 1.857 & 0.083 & 0.762 \\
\hline Finances & 1.9 & 10.0 & 0.155 & 0.055 & 0.436 & -1.862 & $<0.001$ \\
\hline Spirituality and religion & 32.5 & 10.6 & 3.933 & 2.621 & 5.901 & 1.369 & $<0.001$ \\
\hline Health & 14.6 & 36.1 & 0.213 & 0.136 & 0.333 & -1.546 & $<0.001$ \\
\hline Satisfaction & 15.1 & 11.5 & 1.133 & 0.707 & 1.815 & 0.125 & 0.605 \\
\hline Nature and animals & 29.6 & 20.7 & 1.219 & 0.839 & 1.771 & 0.198 & 0.298 \\
\hline Social commitment & 8.3 & 3.9 & 1.941 & 1.015 & 3.715 & 0.663 & 0.045 \\
\hline Hedonism & 7.8 & 8.9 & 0.800 & 0.436 & 1.468 & -0.223 & 0.471 \\
\hline Art and culture & 19.4 & 18.1 & 0.948 & 0.620 & 1.451 & -0.053 & 0.806 \\
\hline Growth & 16.0 & 7.9 & 2.065 & 1.279 & 3.333 & 0.725 & 0.003 \\
\hline
\end{tabular}

Regression models are controlled for sociodemographic variables: age, gender, marital status and education.

MIL, meaning in life. 
Table 4 Linear regression models predicting the subjective QOL among the palliative care patient sample

\begin{tabular}{|c|c|c|c|c|c|c|}
\hline & Coefficient & Robust SE & $\mathrm{T}$ & $P$ value & $95 \% \mathrm{Cl}$ & \\
\hline \multicolumn{7}{|c|}{ Regression from the SMILE scores } \\
\hline los & 0.073 & 0.046 & 1.575 & 0.117 & -0.019 & 0.164 \\
\hline loW & 0.018 & 0.017 & 1.111 & 0.268 & 0.014 & 0.051 \\
\hline loWs & -0.057 & 0.045 & -1.268 & 0.207 & -0.145 & 0.032 \\
\hline \multicolumn{7}{|c|}{ Regression by adding the MIL areas } \\
\hline loW & 0.034 & 0.013 & 2.560 & 0.011 & 0.008 & 0.061 \\
\hline Partnership & 0.932 & 0.515 & 1.810 & 0.072 & -0.084 & 1.949 \\
\hline Home and garden & 1.025 & 0.563 & 1.820 & 0.070 & -0.087 & 2.137 \\
\hline Art and culture & 0.827 & 0.475 & 1.743 & 0.083 & -0.110 & 1.765 \\
\hline Satisfaction & 1.511 & 0.526 & 2.872 & 0.005 & 0.472 & 2.549 \\
\hline Social commitment & 1.814 & 0.644 & 2.815 & 0.005 & 0.542 & 3.086 \\
\hline
\end{tabular}

Regression models are controlled for sociodemographic variables: age, gender, marital status and education; for the backward regression, only significant factors are displayed.

IOS, index of satisfaction; IoW, index of weighting; loWS, index of weighted satisfaction; MIL, meaning in life; QOL, quality of life; SMILE, Schedule for Meaning in Life Evaluation.

Different psychological processes have been described and proposed in the meaning-making model, including, for example, assimilation and accommodation. ${ }^{12}$ These processes contribute to decrease the discrepancy between a global meaning, consistent with the personal sense of identity, and a situational meaning arising in the context of a stressful life event. Such an adaptation or adjustment process may explain the low difference between our two samples. This adjustment hypothesis is plausible because most palliative care patients received their illness diagnosis several months prior to the study, thus allowing for a time frame for adjustment. This hypothesis requires confirmation in a longitudinal study.

\section{Meaning-relevant areas: differences between groups as marks of an adaptation process}

Family and social relations appear to be the most important domains contributing to MIL in both groups, with the addition of 'occupation and work', which is the second most cited area in the Swiss general population. Compared with the general population sample, patients were more likely to cite family and social relations as relational areas, and growth and 'spirituality' as psychoexistential areas.

A relative preponderance of family and social interactions as areas contributing to MIL in palliative care patients was also found in the study of Fegg et al. ${ }^{18}$ Relationships are prominent in other studies exploring the sources of MIL using methodologies

Table 5 Linear regression models predicting the subjective QOL among the Swiss sample

\begin{tabular}{|c|c|c|c|c|c|c|}
\hline & Coefficient & Robust SE & $\mathrm{T}$ & $P$ value & $95 \% \mathrm{Cl}$ & \\
\hline \multicolumn{7}{|c|}{ Regression from the SMILE scores } \\
\hline los & 0.011 & 0.016 & 0.669 & 0.504 & -0.021 & 0.042 \\
\hline loW & 0.017 & 0.004 & 3.932 & 0.000 & 0.009 & 0.026 \\
\hline loWS & 0.030 & 0.016 & 1.904 & 0.057 & -0.001 & 0.061 \\
\hline \multicolumn{7}{|c|}{ Regression by adding the MIL areas } \\
\hline loW & 0.019 & 0.004 & 4.376 & 0.000 & 0.010 & 0.027 \\
\hline loWS & 0.040 & 0.004 & 10.481 & 0.000 & 0.033 & 0.048 \\
\hline Social relation & 0.264 & 0.104 & 2.547 & 0.011 & 0.061 & 0.468 \\
\hline Home and garden & 0.338 & 0.191 & 1.775 & 0.076 & -0.036 & 0.713 \\
\hline Finances & -0.312 & 0.167 & -1.873 & 0.061 & -0.639 & 0.015 \\
\hline Satisfaction & 0.433 & 0.158 & 2.749 & 0.006 & 0.124 & 0.742 \\
\hline Social commitment & 0.466 & 0.252 & 1.847 & 0.065 & -0.029 & 0.962 \\
\hline
\end{tabular}

Regression models are controlled for sociodemographic variables: age, gender, marital status and education; for the backward regression, only significant factors are displayed.

IOS, index of satisfaction; IoW, index of weighting; loWS, index of weighted satisfaction; MIL, meaning in life; QOL, quality of life; SMILE, Schedule for Meaning in Life Evaluation. 
other than the SMILE. ${ }^{31}$ Correspondingly, the social integration dimension was found to be prominent in patients with cancer of different European countries and in India. ${ }^{21} 26$ A systematic review reported the effectiveness of palliative care interventions enhancing social support on the patients' psychological well-being. ${ }^{32}$ Our results also speak in favour of the development of compassionate communities that aim to strengthen this kind of support. ${ }^{33}$

The shift towards more 'inner directed' values and sources, such as spirituality and growth, is noticeable in our study. In the SMILE questionnaire, transcendence is a key element of the spirituality area. It refers to a feeling of connection with an entity that goes beyond our own self. ${ }^{34} 35$ Growth refers to the experiences of self-realisation or self-development. Later stages of life have been shown to lead people towards deeper introspection. ${ }^{36}$ Terror management theory has highlighted the importance of mortality awareness: while being confronted with finitude may induce anxiety, experiential reality of impending death may also elicit a so-called 'mortality-induced growth' associated with a transition from extrinsically oriented goals to intrinsic ones. ${ }^{37}$

\section{Association between MIL and QOL}

The finding that MIL represents a significant predictor of QOL, explaining up to $22 \%$ of the QOL variance, highlights the importance of the existential dimension for QOL in both populations studied and is consistent with previous findings. ${ }^{5}{ }^{17}$ While the association between QOL and sociodemographic variables has been assessed in the general population, ${ }^{38}$ the majority of studies looking at the association of the existential dimension to QOL were realised in the oncology and palliative care settings. ${ }^{4639}$

Interestingly, the part of explained variance increased in the palliative care patients when considering the life areas contributing to MIL (from 13\% to $22 \%$ ), more than in the general population (from $13 \%$ to $15 \%)$. Our results confirm the necessity to focus on the essential life domains in the end-of-life context, in line with the statements mentioned by Frankl in the introduction. These domains may be viewed as relevant to the patients' sense of coherence, allowing them not only to anchor themselves in the reality but also to identify new purposes in which to invest for the remaining time. This approach can also be used to initiate a discussion in order to address the MIL dimension with palliative care patients. Importantly, the use of the SMILE is not restricted to psychologists or chaplains but can encompass the entire healthcare team. In a subsequent step, a referral to a specific professional according to the areas of life mentioned may be appropriate (eg, chaplain, social worker and psychologist).

\section{Limitations}

First, the methodology used to assess MIL with the SMILE questionnaire was different in the two populations, which may account for divergences in the responses. Second, the categories associated with the areas were assigned a posteriori, which implies a degree of subjectivity by the investigators. Third, although a single-item scale for the assessment of QOL appears justified when considering the subjective approach to QOL advocated by the WHO, it does not allow an in-depth assessment of QOL. Concerning the patients, the fact that (i) the participant screening was not systematic in all the palliative care units, (2) only patients with less than 6 months of life expectancy were considered and (3) more than half of the patients who met the inclusion criteria could not participate in the study (which may have led to the exclusion of the most severely affected and unstable patients, not only from a physical but also from an emotional and socioeconomic point of view) contributed to a selection bias. Finally, the crosssectional design of this study does not allow for a causal interpretation of the relationship between MIL and QOL.

\section{CONCLUSIONS}

Our data reinforce the notion of the importance of MIL for QOL in both clinical and non-clinical settings. From a clinical perspective, it highlights the importance of considering closely the concrete life areas contributing to MIL in every single patient, since they play an important role not only for MIL but also for their QOL.

For future research, it will be important to include health status and psychological and social indicators to improve our understanding of the QOL determinants. Different ways of exploring MIL, such as the search for meaning, could help in decreasing the large unexplained MIL variance, and thus contribute to a better understanding of QOL.

Acknowledgements We sincerely thank all the participants of this study for having given their time and their energy for this research.

Contributors $\mathrm{MB}$ drafted the work; participated in the conception and design of the work, and in the acquisition and interpretation of the data; approved the last version; and ensured the accuracy and integrity of any part of the work. $\mathrm{AB}$ participated in the analysis and interpretation of the data, revised it critically, approved the last version, and ensured the accuracy and integrity of any part of the work. FS, CG and GDB participated in the conception and design of the work, revised it critically, approved the last version, and ensured the accuracy and integrity of any part of the work.

Funding We thank the Leenaards Foundation (project number 3548) and the Swiss National Science Foundation (project number 406 740-1 39 313) through the National Research Program 67 'End of Life', which provided financial support for the survey with the representative sample of the Swiss population and the end-of-life patients, respectively. 
Competing interests None declared.

Patient consent for publication Not required.

Ethics approval Regarding the research project involving patients, the human research ethics ethical committee of the Vaud province in Switzerland has approved the project (protocol 182/12). Written consent was obtained from all participants. Regarding the survey with the general Swiss population, the ethical committee did not ask for a submission since the participants were not patients and the data collection was anonymised. Nevertheless, before they orally consented, all participants were informed that this survey was mandated by the University Hospital of Lausanne and concerned perceived MIL, QOL and health status.

Provenance and peer review Not commissioned; externally peer reviewed.

Data availability statement Data are available upon reasonable request. Deidentified data are available upon request from the correspondent author.

Open access This is an open access article distributed in accordance with the Creative Commons Attribution Non Commercial (CC BY-NC 4.0) license, which permits others to distribute, remix, adapt, build upon this work noncommercially, and license their derivative works on different terms, provided the original work is properly cited, appropriate credit is given, any changes made indicated, and the use is noncommercial. See: http://creativecommons.org/licenses/by-nc/4. $0 /$.

\section{ORCID iDs}

Mathieu Bernard http://orcid.org/0000-0003-2823-8806

Claudia Gamondi http://orcid.org/0000-0002-1537-9055

\section{REFERENCES}

1 The WHOQOL group. Development of the world Health organization WHOQOL-BREF quality of life assessment. Psychol Med 1998;28:551-8.

2 Borasio GD, Bernard M. Measure development and assessing outcomes in palliative care: always look on the bright side of life.... Palliat Support Care 2016;14:89-90.

3 Albers G, Echteld MA, de Vet HCW, et al. Evaluation of quality-of-life measures for use in palliative care: a systematic review. Palliat Med 2010;24:17-37.

4 Albers G, Echteld MA, de Vet HCW, et al. Content and spiritual items of quality-of-life instruments appropriate for use in palliative care: a review. J Pain Symptom Manage 2010;40:290-300.

5 Bernard M, Strasser F, Gamondi C, et al. Relationship between spirituality, meaning in life, psychological distress, wish for Hastened death, and their influence on quality of life in palliative care patients. J Pain Symptom Manage 2017;54:514-22.

6 Simmons Z, Bremer BA, Robbins RA, et al. Quality of life in ALS depends on factors other than strength and physical function. Neurology 2000;55:388-92.

7 Nolan SSP, Leget C. Spiritual care in palliative care: working towards an EAPC Task force. European Journal of Palliative Care 2011;18:86-9.

8 Vehling S, Lehmann C, Oechsle K, et al. Global meaning and meaning-related life attitudes: exploring their role in predicting depression, anxiety, and demoralization in cancer patients. Support Care Cancer 2011;19:513-20.

9 Vehling S, Kissane DW, Lo C, et al. The association of demoralization with mental disorders and suicidal ideation in patients with cancer. Cancer 2017;123:3394-401.

10 Rodin G, Lo C, Mikulincer M, et al. Pathways to distress: the multiple determinants of depression, hopelessness, and the desire for hastened death in metastatic cancer patients. Soc Sci Med 2009;68:562-9.
11 Vehling S, Lehmann C, Oechsle K, et al. Is advanced cancer associated with demoralization and lower global meaning? the role of tumor stage and physical problems in explaining existential distress in cancer patients. Psychooncology 2012;21:54-63.

12 Park CL. Making sense of the meaning literature: an integrative review of meaning making and its effects on adjustment to stressful life events. Psychol Bull 2010;136:257-301.

13 Vos J, Vitali D. The effects of psychological meaning-centered therapies on quality of life and psychological stress: a metaanalysis. Palliat Support Care 2018;16:608-32.

14 Vehling S, Philipp R. Existential distress and meaning-focused interventions in cancer survivorship. Curr Opin Support Palliat Care 2018;12:46-51.

15 Frankl V. Man's search for meaning. New-York: Pocket Books, 1976.

16 Fegg MJ, Kramer M, L'hoste S, et al. The schedule for meaning in life evaluation (SMILE): validation of a new instrument for meaning-in-life research. J Pain Symptom Manage 2008;35:356-64.

17 Bernard M, Braunschweig G, Fegg MJ, et al. Meaning in life and perceived quality of life in Switzerland: results of a representative survey in the German, French and Italian regions. Health Qual Life Outcomes 2015;13:160.

18 Fegg MJ, Brandstätter M, Kramer M, et al. Meaning in life in palliative care patients. J Pain Symptom Manage 2010;40:502-9.

19 de Boer AGEM, van Lanschot JJB, Stalmeier PFM, et al. Is a single-item visual analogue scale as valid, reliable and responsive as multi-item scales in measuring quality of life? Qual Life Res 2004;13:311-20.

20 Idler EL, Benyamini Y. Self-Rated health and mortality: a review of twenty-seven community studies. J Health Soc Behav 1997;38:21-37.

21 Tomás-Sábado J, Villavicencio-Chávez C, Monforte-Royo C, et al. What gives meaning in life to patients with advanced cancer? A comparison between Spanish, German, and Swiss patients. J Pain Symptom Manage 2015;50:861-6.

22 Stiefel F, Krenz S, Zdrojewski C, et al. Meaning in life assessed with the "Schedule for Meaning in Life Evaluation" (SMiLE): a comparison between a cancer patient and student sample. Support Care Cancer 2008;16:1151-5.

23 Fegg MJ, Kögler M, Abright C, et al. Meaning in life in patients with progressive supranuclear palsy. Am J Hosp Palliat Care 2014;31:543-7.

24 Fegg MJ, Kögler M, Brandstätter M, et al. Meaning in life in patients with amyotrophic lateral sclerosis. Amyotroph Lateral Scler 2010;11:469-74.

25 Fegg MJ, Kramer M, Bausewein C, et al. Meaning in life in the federal Republic of Germany: results of a representative survey with the schedule for meaning in life evaluation (SMILE). Health Qual Life Outcomes 2007;5:59.

26 Kudla D, Kujur J, Tigga S, et al. Meaning in life experience at the end of life: validation of the Hindi version of the schedule for meaning in life evaluation and a cross-cultural comparison between Indian and German palliative care patients. J Pain Symptom Manage 2015;49:79-88.

27 Mitchell AJ, Chan M, Bhatti H, et al. Prevalence of depression, anxiety, and adjustment disorder in oncological, haematological, and palliative-care settings: a metaanalysis of 94 Interview-Based studies. Lancet Oncol 2011;12:160-74.

28 Rapkin BD, Schwartz CE. Toward a theoretical model of quality-of-life appraisal: implications of findings from studies of response shift. Health Qual Life Outcomes 2004;2:14.

29 Calhoun LG, Tedeschi RG. Posttraumatic growth in clinical practice. London: Routledge, 2013.

30 Windle G. The contribution of resilience to healthy ageing. Perspect Public Health 2012;132:159-60. 
31 Wong PTP, Fry PS. The human quest for meaning: a Handbook of psychological research and clinical application. Mahwah, NJ: Erlbaum, 1998.

32 Bradley N, Lloyd-Williams M, Dowrick C. Effectiveness of palliative care interventions offering social support to people with life-limiting illness-A systematic review. Eur J Cancer Care 2018;27:e12837.

33 Kellehear A. The compassionate city charter: inviting the culturaland social sectors into end-of-life care. In: Wegleitner K, Heimerl K, Kellehear A, eds. Compassionate communities: case studies from Britain and Europe. Abingdon: Routledge, 2016: 76-87.

34 Reed PG. Toward a nursing theory of self-transcendence: deductive reformulation using developmental theories. ANS Adv Nurs Sci 1991;13:64-77.
35 Reed PG. Self-transcendence and mental health in oldest-old adults. Nurs Res 1991;40:5???11-11.

36 Yalom ID. Existential psychotherapy. New York: Basic Books, 1980.

37 Lykins ELB, Segerstrom SC, Averill AJ, et al. Goal shifts following reminders of mortality: reconciling posttraumatic growth and terror management theory. Pers Soc Psychol Bull 2007;33:1088-99.

38 Patrício B, Jesus LMT, Cruice M, et al. Quality of life predictors and normative data. Soc Indic Res 2014;119:1557-70.

39 Cohen SR, Mount BM, Tomas JJ, et al. Existential wellbeing is an important determinant of quality of life. Evidence from the McGill quality of life questionnaire. Cancer 1996;77:576-86. 